\title{
Modeling near-field properties of plasmonic nanoparticles: a surface integral approach
}

\author{
Andreas M. Kern* and Olivier J. F. Martin \\ Nanophotonics and Metrology Laboratory, \\ Swiss Federal Institute of Technology Lausanne (EPFL), 1015 Lausanne, Switzerland
}

\begin{abstract}
Recent developments in nanofabrication and optical near-field metrology have faced complementary modeling techniques with new demands. We present a surface integral formulation that accurately describes the extreme near-field of a plasmonic nanoparticle in addition to its far-field properties. Flexible surface meshing gives precise control over even complex geometries allowing investigation of the effects of fabrication accuracy and material homogeneity on a particle's optical response. Using this technique, the influence of a particle's symmetry and shape on surrounding "hot spots" of extremely large field enhancement is explored, giving insight into the mechanisms of surface enhanced Raman scattering and single-molecule detection techniques.
\end{abstract}

\section{INTRODUCTION}

As modern optical measurement techniques are becoming more and more advanced, they pose increasingly high demands on accompanying simulation routines. In turn, a wide range of approaches has been developed for the simulation of the interaction of electromagnetic radiation with matter. Each approach, however, has its region of application and may be unsuitable under other conditions. While some approaches intrinsically provide far-field characteristics of a simulated system, for example, they may be unable to reproduce the field distribution close to the scattering objects. Approaches that offer the possibility to simulate the properties of very inhomogeneous materials may suffer from inflexible discretization, restricting geometric versatility.

First developed for the simulation of interstellar dust, ${ }^{1-3}$ numerical scattering routines based in integral equations (IE) have become popular approches for simulating the optical properties of man-made nanoparticles. ${ }^{4-10}$ Two well-known implementations of this approach include the discrete dipole approximation ${ }^{3}$ (DDA) and the Green's tensor method. ${ }^{11}$ While these methods are powerful as they require discretization of the scatterer only and not the surrounding space, they suffer from poor scaling and inflexible meshing caused by the required volume discretization. Much can be gained, however, if one can reduce the number of discretization elements in an IE simulation without losing accuracy.

To this end, we investigate in this paper an approach based on the transformation of the volume integral equation (VIE) to a surface integral equation (SIE) formulation. ${ }^{12}$ This procedure, while quite well-known in microwave studies, has only rarely been applied in optics. ${ }^{13-18}$ In the first part of this paper we briefly introduce the most popular electromagnetic simulation routines, showing their range of application as well as their deficits. Next we show the main steps in deriving the proposed SIE formulation, including important post-processing procedures. Finally, we demonstrate the capacity of this method, including the calculation of extreme near-field properties of realistic nanoparticles.

\section{SURFACE INTEGRAL EQUATION FORMULATION}

In this section, the surface integral equation formulation will be introduced and its relation to other routines for electromagnetic simulation will be given.

*andreas.kern@epfl.ch

Plasmonics: Nanoimaging, Nanofabrication, and their Applications $\mathrm{V}$, edited by Satoshi Kawata, Vladimir M. Shalaev, Din Ping Tsai, Proc. of SPIE Vol. 7395

739518 - @ 2009 SPIE · CCC code: 0277-786X/09/\$18 - doi: 10.1117/12.825833

Proc. of SPIE Vol. 7395 739518-1 
(a)

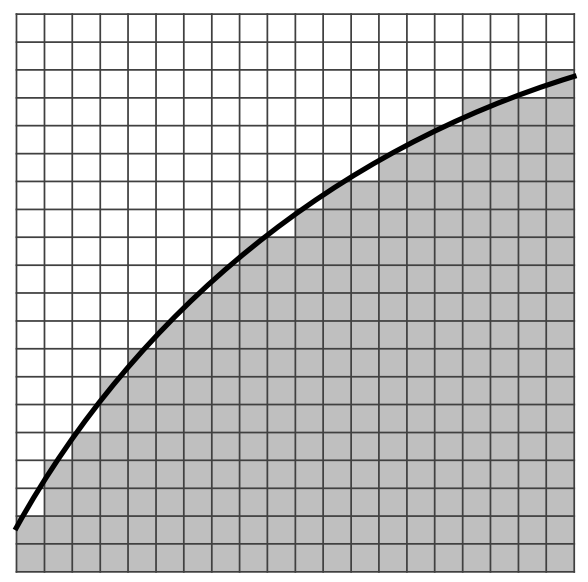

(b)

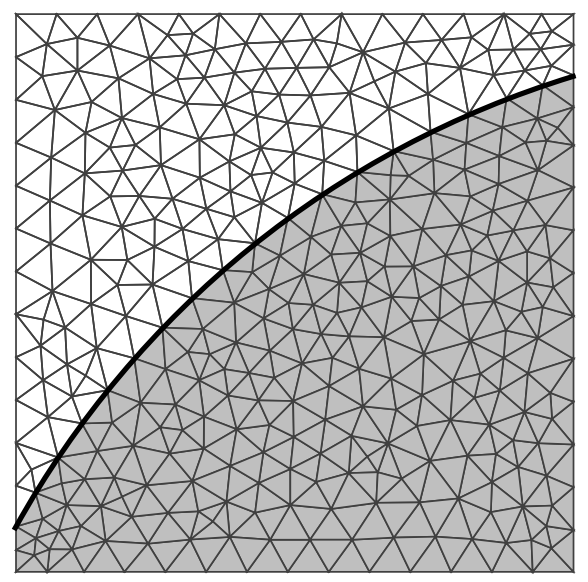

Figure 1. Approximation of a curved structure (black line) by (a) a structured grid and (b) an unstructured mesh. Method (b) clearly shows a better approximation, even with a similar number of discretization elements.

\subsection{Overview of electromagnetic simulation routines}

The physical background of most electromagnetic simulation routines including the SIE formulation lies in solving Maxwell's equations for given boundary conditions. In a differential form, these are given by

$$
\begin{aligned}
& \nabla \times \mathbf{H}=\frac{\partial \mathbf{D}}{\partial t}+\mathbf{j} \\
& \nabla \times \mathbf{E}=-\frac{\partial \mathbf{B}}{\partial t}
\end{aligned}
$$

\subsubsection{Finite-difference time-domain method}

Equations (1) are directly the starting point for the finite-differente time-domain (FDTD) method. ${ }^{19,20}$ In this method, discrete representations of the above differential equations are derived using difference quotients in the place of the continuous derivatives. These discrete representations can then be used as update equations, allowing to calculate the time development of an electromagnetic field distribution given its initial value.

The update equations are typically given in cartesian coordinates requiring difference quotients to be calculated in $x$ - $y$ - and $z$-directions. A method for storing the field distributions has been proposed for highly efficient calculation of the required difference quotients. ${ }^{19}$ In this method, however, one is in general limited to orthogonal meshes in which neighboring mesh elements lie along the $x$ - $y$ - and $z$-axes. While the grid size need not be homogeneous, effective mesh refinement is strongly limited, large refinement factors usually requiring subgridding. ${ }^{21,22}$ In addition, material constants such as electrical permittivity are applied to each grid mesh, allowing for the simulation of highly inhomogeneous systems but introducing discretization errors due to staircasing (Fig. 1 (a), especially for rounded objects.

\subsubsection{Finite element method}

The finite element method (FEM) is a versatile approach capable of approximating solutions to a wide range of partial differential equations (PDEs). In electromagnetics ${ }^{23}$ it is usually used to solve the Helmhotz equations,

$$
\begin{aligned}
\nabla^{2} \mathbf{E}+k^{2} \mathbf{E} & =0, \\
\nabla^{2} \mathbf{H}+k^{2} \mathbf{H} & =0,
\end{aligned}
$$

where $k$ is the complex wavenumber. These can be derived from Eqs. (1) assuming a harmonic time development of the electromagnetic fields. A characteristic of the FEM is the application of Galerkin's method, in which the 
same functions (generally tent functions) are used as basis functions to approximate the solution of the PDE and as testing functions to obtain a discrete system of equations. This approach allows for a more flexible discretization as the grid needs neither be structured nor homogeneous, enabling efficient mesh refinement and more accurate approximation of rounded objects (Fig. 1(b).

A drawback of both FDTD and FEM methods is the need to discretize not only the desired object itself, but also the space surrounding it. This is because the incident light is usually modeled as an emissive media or an initial condition inside the discretization grid. In addition, as both methods use a mesh element's neighbors to calculate the field value of the element itself, special care must be taken at the edges of the discretized domain, where special boundary conditions are applied. The most common boundary conditions are periodic, reflecting and absorbing ${ }^{24,25}$ boundary conditions, the latter allowing the simulation of a particle in an effectively infinite background. Most of these boundary conditions are, however, most easily defined on straight boundaries, demanding the discretization of a rectangular area or volume around the object.

\subsubsection{Volume integral equation}

The volume integral equation (VIE) formulation is based on the Green's function formalism in which the knowledge of a solution to a simple form of a PDE can be used to solve a more complicated one. Consider the inhomogeneous scalar PDE

$$
\left(\nabla^{2}-k^{2}(\mathbf{r})\right) \psi(\mathbf{r})=s(\mathbf{r}),
$$

which corresponds to the scalar Helmhotz equation with an arbitrary scalar source distribution $s(\mathbf{r})$. One now defines the Green's function $G$ as solving the special case

$$
\left(\nabla^{2}-k^{2}(\mathbf{r})\right) G\left(\mathbf{r}, \mathbf{r}^{\prime}\right)=-\delta\left(\mathbf{r}-\mathbf{r}^{\prime}\right),
$$

with a single point source located at $\mathbf{r}^{\prime}$. Multiplying both sides of Eq. (4) with $s(\mathbf{r})$ and integrating over $\mathbf{r}^{\prime}$ one obtains

$$
\left(\nabla^{2}+k^{2}(\mathbf{r})\right)\left[-\int_{\mathbb{R}^{3}} \mathrm{~d} \mathbf{r}^{\prime} s\left(\mathbf{r}^{\prime}\right) G\left(\mathbf{r}, \mathbf{r}^{\prime}\right)\right]=\int_{\mathbb{R}^{3}} \mathrm{~d} \mathbf{r}^{\prime} s\left(\mathbf{r}^{\prime}\right) \delta\left(\mathbf{r}-\mathbf{r}^{\prime}\right) .
$$

Recognizing the right-hand side of Eq. (5) as $s(\mathbf{r})$ and comparing to Eq. (3) one can see that

$$
\psi(\mathbf{r})=-\int_{V} \mathrm{~d} \mathbf{r}^{\prime} s\left(\mathbf{r}^{\prime}\right) G\left(\mathbf{r}, \mathbf{r}^{\prime}\right),
$$

where $V$ is the support of $s$. The source distribution $s$ causes a deviation to regular wave propagation according to $k(\mathbf{r})$ and thus descibes the simulated object while $k$ describes the background in which the object is located. One can see that in the Green's formalism the solution $\psi$ can be determined anywhere in space by integrating over the object only, thus there is no need to discretize the space around the object or to introduce absorbing boundary conditions to simulate an object in free space.

The Green's function $G$ can be found for a number of distributions of $k(\mathbf{r})$. For homogeneous media $k(\mathbf{r})=$ $k=$ const, for example,

$$
G\left(\mathbf{r}, \mathbf{r}^{\prime}\right)=\frac{\exp \left(i k\left|\mathbf{r}-\mathbf{r}^{\prime}\right|\right)}{4 \pi\left|\mathbf{r}-\mathbf{r}^{\prime}\right|},
$$

but solutions can also be derived for stratified media, ${ }^{26}$ for example. In the case of three-dimensional electromagnetics simulations, the PDE to be solved is no longer scalar but vectorial. The Green's formalism, however, can be similarly applied, the obtained Green's functions no longer scalars but tensors.

The main advantage of VIE formulations is the fact that only the scattering object itself needs to be discretized, the response of the background being completely described by the Green's function. In this fashion, the properties of a single scatterer on an infinitely large substrate, for example, can be simulated. The material properties of the scatterer, such as its permittivity, can vary from one discretization element to another allowing the simulation of inhomogeneous scatterers.

In most cases, however, one is limited to very simple discretization elements such as cubes or spheres, leading to staircasing like in the FDTD method, albeit less pronounced as the element sizes can be varied to better 


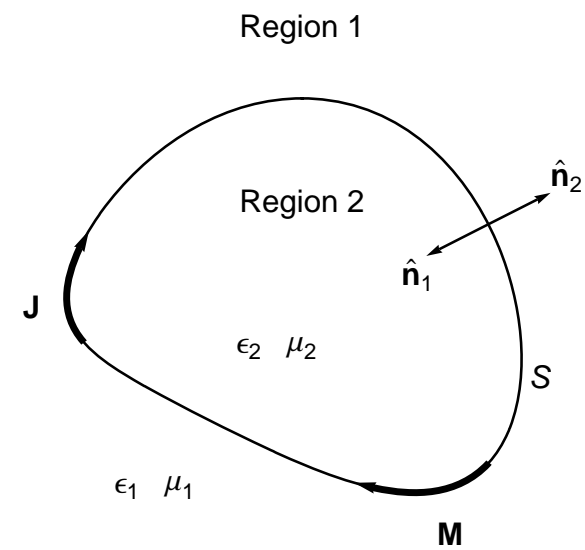

Figure 2. Geometry of the considered two-region problem.

approximate i.e. a curved surface. Also, as the fields in the VIE formulation are not calculated locally but globally using the system's Green's function, the matrices produced are dense, while those produced in the FEM method, for example, are sparse. This leads to a poor scaling of the VIE formulation, making it less applicable for electrically large problems.

\subsection{Surface integral equation}

The surface integral equation (SIE) method is an approach quite common in microwave studies but rarely used in the optical regime. ${ }^{16-18}$ The principle behind it is the transformation of volume integrals like the one in Eq. (6) to surface integrals using Gauss' theorem. Besides reducing scaling from the third to the second power, this approach offers many advantages as will be shown in this section.

\subsubsection{Derivation}

Consider a two-region geometry as shown in Fig. 2. We assume harmonic electric and magnetic fields of the form $\mathbf{U}_{i}=\mathbf{U}_{0, i} \exp (-i \omega t)$ in both regions $i=1,2$. To solve Maxwell's equations, the electric field $\mathbf{E}_{i}$ in each region must satisfy the equation

$$
\nabla \times \nabla \times \mathbf{E}_{i}(\mathbf{r})-k_{i}^{2} \mathbf{E}_{i}(\mathbf{r})=i \omega \mu_{i} \mathbf{j}(\mathbf{r}), \quad \mathbf{r} \in V_{i}
$$

where $k_{i}=\left(\omega^{2} \epsilon_{i} \mu_{i}\right)^{1 / 2}$ is the wave number for electromagnetic waves in region $i$ and $\mathbf{j}$ denotes a volume current density. Similar to the scalar case in Eq. (4), a dyadic Green's function $\overline{\mathbf{G}}_{i}$ for each region is defined as solving

$$
\nabla \times \nabla \times \overline{\mathbf{G}}_{i}\left(\mathbf{r}, \mathbf{r}^{\prime}\right)-k_{i}^{2} \overline{\mathbf{G}}_{i}\left(\mathbf{r}, \mathbf{r}^{\prime}\right)=\overline{\mathbf{1}} \delta\left(\mathbf{r}-\mathbf{r}^{\prime}\right) .
$$

Combining Eqs. (8) and (9), integrating over $\mathbf{r}$ and applying the Green-Gauss theorem, ${ }^{12,27}$ we can derive the electric field integral equation (EFIE),

$$
\begin{aligned}
& \left(\frac{\omega \mu_{i}}{i} \int_{S} \mathrm{~d} S^{\prime} \overline{\mathbf{G}}_{i}\left(\mathbf{r}, \mathbf{r}^{\prime}\right) \cdot \mathbf{J}\left(\mathbf{r}^{\prime}\right)-\int_{S} \mathrm{~d} S^{\prime}\left[\nabla^{\prime} \times \overline{\mathbf{G}}_{i}\left(\mathbf{r}, \mathbf{r}^{\prime}\right)\right] \cdot \mathbf{M}\left(\mathbf{r}^{\prime}\right)\right)_{\tan } \\
& =\left\{\begin{array}{lll}
\left(\mathbf{E}_{1}^{\mathrm{inc}}(\mathbf{r})\right)_{\tan }: & i=1 \\
0 & : & i=2
\end{array}\right.
\end{aligned}
$$

for $\mathbf{r} \in S$, where the subscript tan denotes tangential components, $\mathbf{E}^{\text {inc }}$ is the incident electrical field and $\mathbf{J}=\hat{\mathbf{n}}_{2} \times \mathbf{H}_{i}$ and $\mathbf{M}=-\hat{\mathbf{n}}_{2} \times \mathbf{E}_{i}$ are equivalent electric and magnetic surface currents on the surface of the scatterer. Similarly, starting from the magnetic field equivalent of Eq. (8), one can derive the magnetic field integral equation (MFIE),

$$
\begin{aligned}
& \left(\frac{\omega \epsilon_{i}}{i} \int_{S} \mathrm{~d} S^{\prime} \overline{\mathbf{G}}_{i}\left(\mathbf{r}, \mathbf{r}^{\prime}\right) \cdot \mathbf{M}\left(\mathbf{r}^{\prime}\right)+\int_{S} \mathrm{~d} S^{\prime}\left[\nabla^{\prime} \times \overline{\mathbf{G}}_{i}\left(\mathbf{r}, \mathbf{r}^{\prime}\right)\right] \cdot \mathbf{J}\left(\mathbf{r}^{\prime}\right)\right)_{\tan } \\
& =\left\{\begin{array}{lll}
\left(\mathbf{H}_{1}^{\mathrm{inc}}(\mathbf{r})\right)_{\tan }: & i=1 \\
0 & : & i=2
\end{array}\right.
\end{aligned}
$$




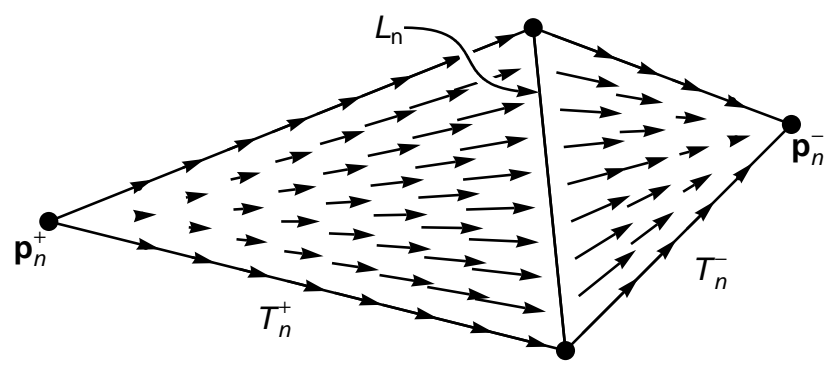

Figure 3. RWG basis function $\mathbf{f}_{n}$ is nonzero on the two adjacent triangles $T_{n}^{+}$and $T_{n}^{-}$and zero everywhere else. The normal component of $\mathbf{f}_{n}$ is continuous across all edges, i.e. zero on all outer edges.

for $\mathbf{r} \in S$. One can see that in Eqs. (10) and (11), only surface integrals appear, giving this method its name.

An approximate solution to the EFIE on a discretized surface can be found using the method of moments ${ }^{28}$ (MoM). First, the unknown surface currents $\mathbf{J}$ and $\mathbf{M}$ are expanded using vectorial basis functions,

$$
\begin{aligned}
\mathbf{J}(\mathbf{r}) & =\sum_{n=1}^{N} \alpha_{n} \mathbf{f}_{n}(\mathbf{r}) \\
\mathbf{M}(\mathbf{r}) & =\sum_{n=1}^{N} \beta_{n} \mathbf{f}_{n}(\mathbf{r}) .
\end{aligned}
$$

The most popular basis functions on a triangular mesh are RWG functions, ${ }^{29}$ named after their inventors. For a pair of adjacent triangles $T_{n}^{+}$and $T_{n}^{-}$sharing a common edge $n$, these are defined by

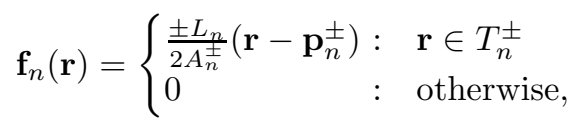

where $L_{n}$ is the length of the common edge, $A_{n}^{ \pm}$denotes the areas and $\mathbf{p}_{n}^{ \pm}$the free vertices of the triangles on either side (Fig. 3). Similarly to the FEM method, the Galerkin method is now applied, multiplying Eq. (10) with the basis functions and integrating over $S$, resulting in

$$
\begin{aligned}
& \int_{S_{m}} \mathrm{~d} S \mathbf{f}_{m}(\mathbf{r}) \cdot \sum_{n=1}^{N}\left(\alpha_{n} \frac{\omega \mu_{i}}{i} \int_{S_{n}} \mathrm{~d} S^{\prime} \overline{\mathbf{G}}_{i}\left(\mathbf{r}, \mathbf{r}^{\prime}\right) \cdot \mathbf{f}_{n}\left(\mathbf{r}^{\prime}\right)-\beta_{n} \int_{S_{n}} \mathrm{~d} S^{\prime}\left[\nabla^{\prime} \times \overline{\mathbf{G}}_{i}\left(\mathbf{r}, \mathbf{r}^{\prime}\right)\right] \cdot \mathbf{f}_{n}\left(\mathbf{r}^{\prime}\right)\right)
\end{aligned}
$$

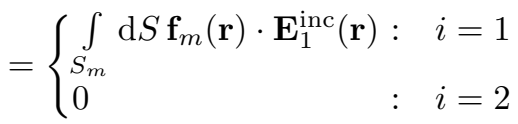

for all $m=1 \ldots N$, where $S_{m}=T_{m}^{+} \cup T_{m}^{-}$is the support of basis function $\mathbf{f}_{m}$. This equation can be written in a matrix form,

$$
\mathbf{K} \cdot \boldsymbol{\psi}=\mathbf{q},
$$

with vectors $\boldsymbol{\psi}$ and $\mathbf{q}$ and matrix $\mathbf{K}$. Here, $\boldsymbol{\psi}$ contains the unknowns $\alpha_{n}$ and $\beta_{n}$, $\mathbf{q}$ contains the incident field values and $\mathbf{K}$ contains the remaining integrals, embodying the geometric and optical properties of the scatterer.

A similar matrix equation can be set up for the MFIE, starting with Eq. (11). However, solving for $\boldsymbol{\psi}$ will in some cases not result in the same values for $\alpha_{n}, \beta_{n}$ as with the EFIE. In fact, especially in resonant conditions, solutions to the EFIE or MFIE alone may exhibit large errors due to poor testing. A remedy to this problem is to solve both EFIE and MFIE simultaneously, assuming an identical solution $\boldsymbol{\psi}$. Various combinations have been proposed and their reliability is the subject of ongoing research. ${ }^{30-32}$ One combination which has proved to provide stable and accurate results even in resonant conditions is the PMCHW formulation, named after its inventors. ${ }^{30,33-36}$ 


\subsubsection{Singularity of Green's functions}

To calculate the matrix elements $K_{m n}$, one must first find an expression for the dyadic Green's function $\overline{\mathbf{G}}$. For homogeneous bodies, one can choose the Green's function for unbounded homogeneous media, ${ }^{4,27}$ given by

$$
\overline{\mathbf{G}}_{i}\left(\mathbf{r}, \mathbf{r}^{\prime}\right)=\left(\overline{\mathbf{1}}+\frac{\nabla \nabla}{k_{i}^{2}}\right) \frac{\exp \left(i k_{i}\left|\mathbf{r}-\mathbf{r}^{\prime}\right|\right)}{4 \pi\left|\mathbf{r}-\mathbf{r}^{\prime}\right|}=\left(\overline{\mathbf{1}}+\frac{\nabla \nabla}{k_{i}^{2}}\right) G_{i}\left(\mathbf{r}, \mathbf{r}^{\prime}\right) .
$$

One can see that Eq. (16) is singular for $\mathbf{r}=\mathbf{r}^{\prime}$ and as Eq. (14) contains double integrals over $\overline{\mathbf{G}}$, the integrands will diverge if the integration areas $S_{m}$ and $S_{n}$ overlap. An elegant way to compute these integrals nonetheless as well as improve numerical accuracy for $S_{m}$ near $S_{n}$ is to extract the singularity from the Green's function, ${ }^{37-40}$ obtaining a singular but analytically simple part and a slowly changing part that can accurately be integrated numerically e.g. by Gaussian quadrature. ${ }^{41}$

Observe the Taylor expansion of $G_{i}\left(\mathbf{r}, \mathbf{r}^{\prime}\right)$,

$$
G_{i}\left(\mathbf{r}, \mathbf{r}^{\prime}\right)=\frac{1}{4 \pi}\left(\frac{1}{R}+i k_{i}-\frac{k_{i}^{2} R}{2}+\ldots\right), \quad R=\left|\mathbf{r}-\mathbf{r}^{\prime}\right| .
$$

Subtracting from $G_{i}$ the first two odd terms yields a smoothed Green's function $G_{i}^{S}$ which is non-singular and differentiable for $R=0$,

$$
G_{i}\left(\mathbf{r}, \mathbf{r}^{\prime}\right)=\underbrace{\frac{\exp \left(i k_{i} R\right)}{4 \pi R}-\frac{1}{4 \pi}\left(\frac{1}{R}-\frac{k_{i}^{2} R}{2}\right)}_{G_{i}^{S}\left(\mathbf{r}, \mathbf{r}^{\prime}\right)}+\frac{1}{4 \pi}\left(\frac{1}{R}-\frac{k_{i}^{2} R}{2}\right) .
$$

Performing this separation on the Green's functions in Eq. (14), the integrals can be split into a sum of two integrals, one which can accurately solved numerically and an integral over the subtracted Taylor terms. The latter can be expressed using the integrals

$$
\begin{aligned}
K_{1}^{q}(T) & =\int_{T} R^{q} \nabla^{\prime} \cdot \mathbf{f}_{n}\left(\mathbf{r}^{\prime}\right) \mathrm{d} S^{\prime}, \\
\mathbf{K}_{2}^{q}(T) & =\int_{T} R^{q} \mathbf{f}_{n}\left(\mathbf{r}^{\prime}\right) \mathrm{d} S^{\prime}, \\
\mathbf{K}_{3}^{q}(T) & =\int_{T} \nabla^{\prime} R^{q} \mathrm{~d} S^{\prime}, \\
\mathbf{K}_{4}^{q}(T) & =\int_{T}\left(\nabla^{\prime} R^{q}\right) \times \mathbf{f}_{n}\left(\mathbf{r}^{\prime}\right) \mathrm{d} S^{\prime},
\end{aligned}
$$

for which closed-form solutions can be given for RWG functions $\mathbf{f}_{n}$ and triangular surfaces $T .{ }^{39}$ In this manner, Eq. (14) can be solved accurately even for close and overlapping $S_{m}, S_{n}$. As will be shown in this paper, however, the singularity subtraction technique can also be used to improve field calculation and excitation accuracies.

\subsubsection{Calculation of field distributions}

The surface currents $\mathbf{J}$ and $\mathbf{M}$ are not the actual currents flowing on the simulated object. These equivalent currents, however, flowing on the conductive, hollow shell of the object, produce the same electromagnetic field inside and around the object as the object itself. Thus, to calculate the fields, one must only take into account the field generated by the surface currents and one can derive an expression for the electric field at the observation point $\mathbf{r},{ }^{12}$

$$
\begin{aligned}
& \mathbf{E}_{i}(\mathbf{r})=\left\{\begin{array}{l}
+ \\
-
\end{array}\right\} \sum_{n}\left[-\alpha_{n} \frac{\omega \mu_{i}}{i} \int_{S_{n}} \mathrm{~d} S^{\prime} \overline{\mathbf{G}}_{i}\left(\mathbf{r}, \mathbf{r}^{\prime}\right) \cdot \mathbf{f}_{n}\left(\mathbf{r}^{\prime}\right)+\beta_{n} \int_{S_{n}} \mathrm{~d} S^{\prime}\left[\nabla^{\prime} G_{i}\left(\mathbf{r}, \mathbf{r}^{\prime}\right)\right] \times \mathbf{f}_{n}\left(\mathbf{r}^{\prime}\right)\right]
\end{aligned}
$$

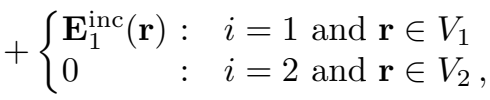


while for the magnetic field one obtains

$$
\begin{aligned}
& \mathbf{H}_{i}(\mathbf{r})=\left\{\begin{array}{l}
+ \\
-
\end{array}\right\} \sum_{n}\left[-\beta_{n} \frac{\omega \epsilon_{i}}{i} \int_{S_{n}} \mathrm{~d} S^{\prime} \overline{\mathbf{G}}_{i}\left(\mathbf{r}, \mathbf{r}^{\prime}\right) \cdot \mathbf{f}_{n}\left(\mathbf{r}^{\prime}\right)-\alpha_{n} \int_{S_{n}} \mathrm{~d} S^{\prime}\left[\nabla^{\prime} G_{i}\left(\mathbf{r}, \mathbf{r}^{\prime}\right)\right] \times \mathbf{f}_{n}\left(\mathbf{r}^{\prime}\right)\right]
\end{aligned}
$$

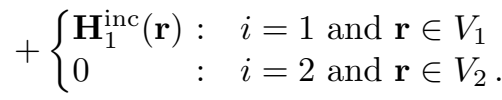

One can see that as the observation point $\mathbf{r}$ approaches the surface of the object, the singularity of the Green's function becomes problematic. The singularity subtraction method explained in Sec. 2.2.2, however, can again be used to accurately calcuate the integrals. Though for points $\mathbf{r}$ not touching the surface, the integrals are not strictly singular, they are very difficult to calculate numerically and the singularity subtraction technique can greatly improve accuracy.

\subsubsection{Illumination}

In the SIE method, illumination is defined by the fields $\mathbf{E}^{\text {inc }}(\mathbf{r})$ and $\mathbf{H}^{\text {inc }}(\mathbf{r})$, contained in the integrals in the right-hand side of Eq. (14). These fields must solve Maxwell's equations for the given background for which the Green's function is valid. In many free-space simulations, plane waves are used as illumination, but another interesting configuration is illumination by a single dipole source. The electric and magnetic fields of an electric dipole are given by

$$
\begin{aligned}
\mathbf{E}_{d}\left(\mathbf{r}, \mathbf{r}^{\prime}\right) & =i k Z_{0} \mathbf{G}\left(\mathbf{r}, \mathbf{r}^{\prime}\right) \cdot \mathbf{p}, \\
\mathbf{H}_{d}\left(\mathbf{r}, \mathbf{r}^{\prime}\right) & =-\nabla G\left(\mathbf{r}, \mathbf{r}^{\prime}\right) \times \mathbf{p},
\end{aligned}
$$

where $\mathbf{r}$ is the observation point, $\mathbf{r}^{\prime}$ is the location of the dipole and $\mathbf{p}$ is orientation of the dipole. Taking $\mathbf{E}_{d}$ and $\mathbf{H}_{d}$ as incident fields, one can again see that if $\mathbf{r}^{\prime}$ comes very close to the surface, the integrals on the right-hand side of Eq. (14) become problematic. This is yet another application of the singularity subtraction technique described in Sec. 2.2.2. Using this technique, a structure can be illuminated by a dipole arbitrarily close to its surface.

\subsection{Post processing}

Once the coefficients $\alpha_{n}, \beta_{n}$ and therewith the surface currents $\mathbf{J}$ and $\mathbf{M}$ have been determined, the electromagnetic field can be calculated at any point in space using Eqs. (23) and (24). To be able to compare simulation results with experimental observations, one is often faced with the task of computing further attributes of the simulated system such as its optical cross sections. ${ }^{42}$ The capability of the SIE method to accurately compute the electromagnetic fields both far from and close to the simulated structure greatly facilitates determination of cross sections as well as descriptive field distributions.

\subsubsection{Cross section calculation}

The bistatic scattering cross section of a particle can easily be computed using

$$
\sigma_{\mathrm{sca}}(\theta, \varphi)=4 \pi R^{2} \frac{\left|\mathbf{E}_{\mathrm{sca}}(\mathbf{r}(\theta, \varphi))\right|^{2}}{\left|\mathbf{E}_{\mathrm{inc}}\right|^{2}},
$$

where $\mathbf{r}(\theta, \varphi)$ is a vector a distance $R$ from the center of the scatterer at inclination and azimuthal angles $\theta$ and $\varphi$ from the incident plane wave's propagation direction. The scattered field $\mathbf{E}_{\mathrm{sca}}=\mathbf{E}_{1}-\mathbf{E}_{1}^{\text {inc }}$ outside of the sphere is given by Eq. (23) without the last term.

An elegant way of determining the total scattering as well as extinction and absorption cross sections from a system modeled with a surface integral formulation is via the Poynting vector,

$$
\mathbf{S}(\mathbf{r})=\frac{1}{2} \operatorname{Re}\left\{\mathbf{E}(\mathbf{r}) \times \mathbf{H}^{*}(\mathbf{r})\right\} .
$$



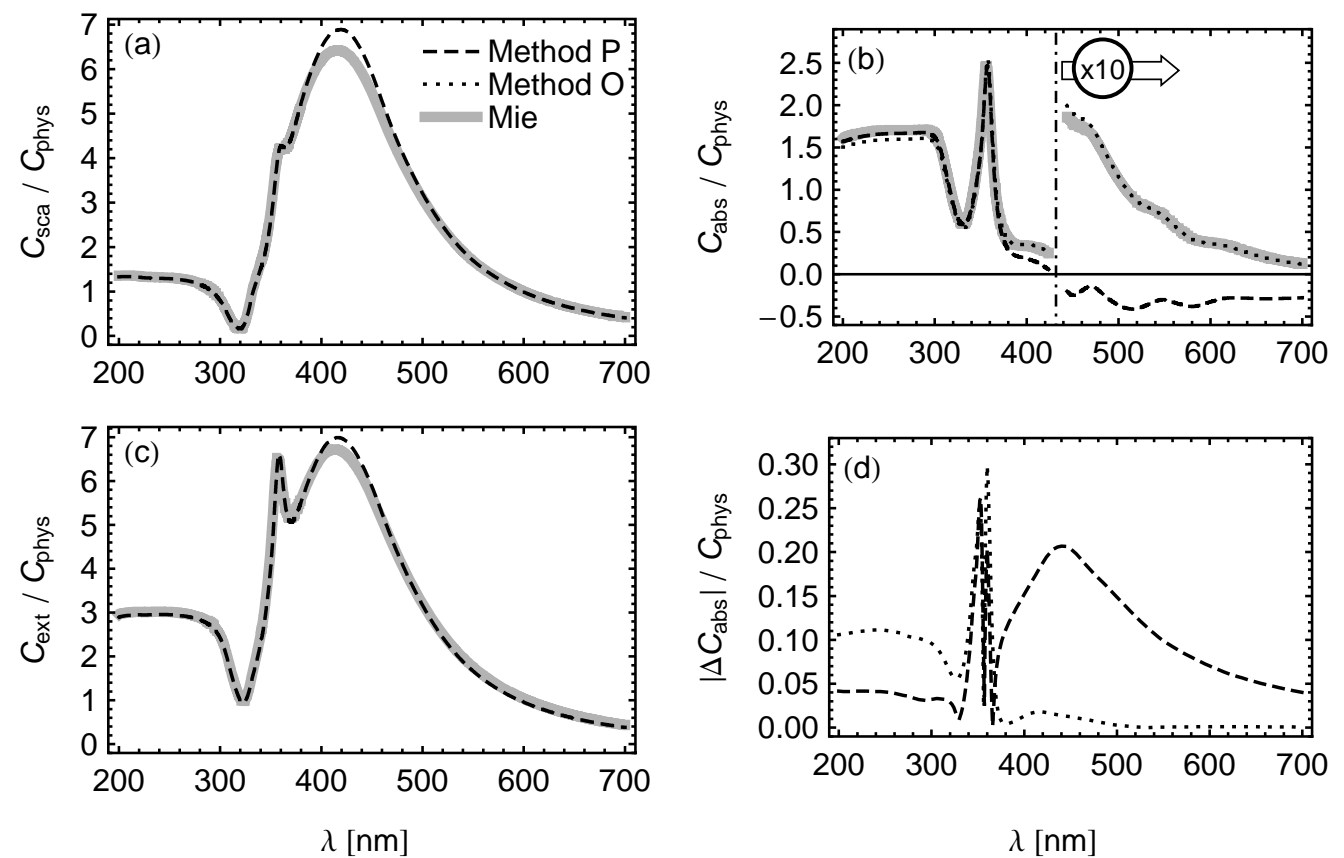

Figure 4. Scattering, absorption and extinction cross sections [(a), (b) and (c), respectively] of a silver sphere (radius $r=60 \mathrm{~nm}$ ) obtained by Poynting vector integration (method P) and Ohmic loss (method O) compared to the exact Mie solution. Note that scattering and extinction cross sections cannot be determined using method $\mathrm{O}$ alone. The absolute value of the error of the absorption cross section is shown in (d). All values are given relative to the physical cross section $C_{\text {phys }}=\pi r^{2}$ of the sphere.

The absorption cross section $C_{a}$ can immediately be determined as

$$
C_{a}=-\frac{1}{I_{\mathrm{inc}}} \int_{A} \mathrm{~d} \mathbf{A} \cdot \mathbf{S}(\mathbf{r})
$$

where $I_{\text {inc }}$ is the incident intensity and $A$ is an arbitrary closed surface completely containing the scatterer. Separating the fields into incident and scattered components, the Poynting vector can be split into three components (see Ref. 42, Sec. 3.3),

$$
\mathbf{S}(\mathbf{r})=\mathbf{S}_{\text {inc }}(\mathbf{r})+\mathbf{S}_{\text {scat }}(\mathbf{r})+\mathbf{S}_{\text {ext }}(\mathbf{r})
$$

allowing the computation of scattering and extinction cross sections analogously to Eq. 29. This approach is advantageous because it requires no further discretization of the scatterer, only the numerical integration of the known distributions $\mathbf{S}$.

As an example, the optical response of a silver sphere of radius $r=60 \mathrm{~nm}$ illuminated by a plane wave of wavelength $\lambda$ was simulated. The cross sections obtained via the Poynting vector are shown as dashed lines in Fig. 4. One can see that while scattering and extinction cross sections are in excellent agreement with the exact Mie solution ${ }^{42,43}$ (thick gray line), the absorption cross section displays a large relative error for longer wavelengths, even becoming unphysically negative. This is caused by the numerical integration required by this method in which many large positive and negative values are added together, mostly canceling each other out. This results in a comparably large error of $C_{\mathrm{abs}}$, permitting even negative values.

To increase the accuracy of the calculated absorption cross section, a different approach can be chosen: the absorption can also be determined via the Ohmic loss in the scatterer. In this method, the absorption cross section is given by

$$
C_{\text {abs }}=\frac{1}{2 I_{\text {inc }}} \int_{\Omega} \mathrm{d} V \operatorname{Re}\{\sigma\}|\mathbf{E}(\mathbf{r})|^{2},
$$

where $\Omega$ is the volume of the scatterer and $\sigma$ is its conductivity. As shown by the dotted lines in Fig. 4, this approach ensures a nearly constant relative error of $C_{\text {abs }}$, completely barring negative values. Unfortunately, 
(a)

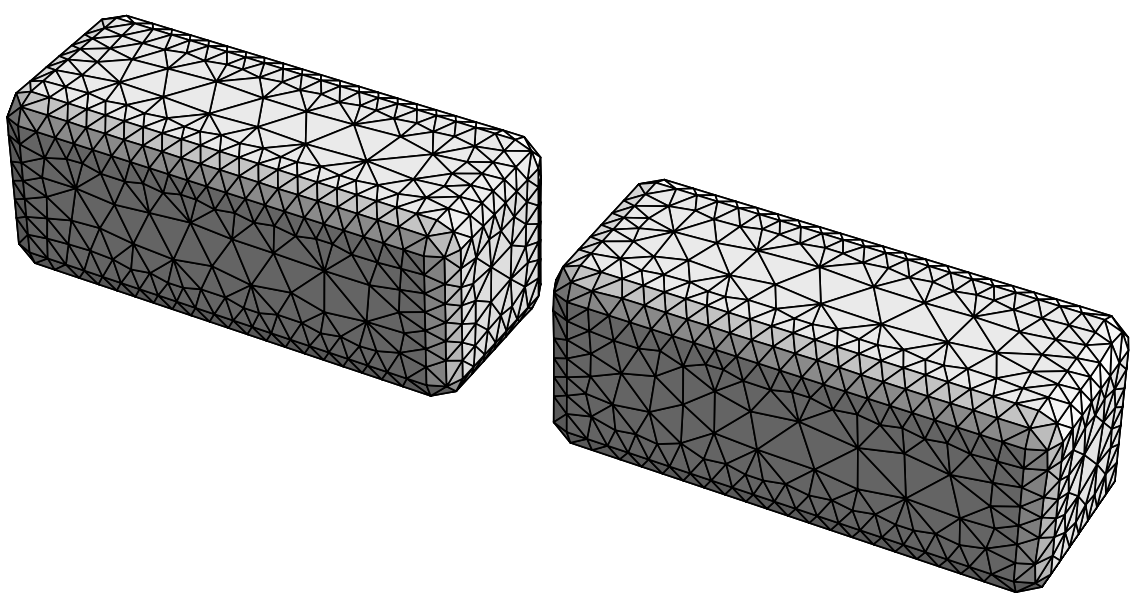

(b)

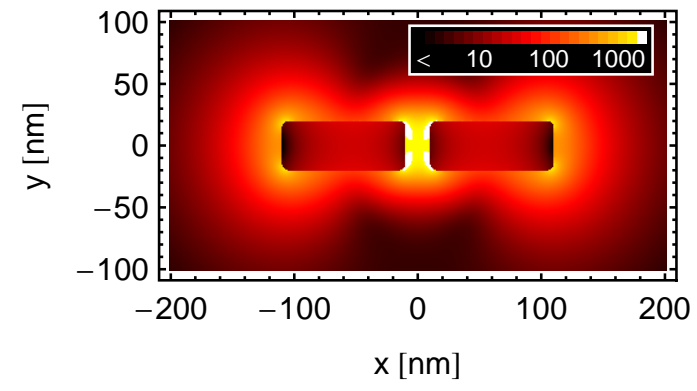

(c)

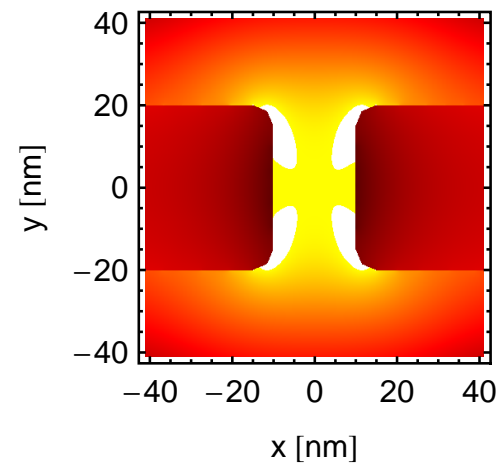

Figure 5. Simulation of a resonantly excited nano-antenna. Subfigure (a) shows the discretization mesh, subfigures (b) and (c) show the intensity distribution on planes through the center of the antenna's arms. Illumination is incident along $z$ (out of the image planes), polarized along $x$ with an intensity of 1 . White areas are clipped.

this method requires a volume integral of the scatterer. This can be done by either discretizing the scatterer into elements of known volume or by a more statistical approach, in which points are randomly distributed in and around the scatterer with a known mean separation $\bar{s}$. Using a binary space partitioning (BSP) tree, ${ }^{44}$ one can quickly determine if a given point is inside or outside of the surface mesh, then summing over all the points inside the scatterer multiplied by $\bar{s}^{3}$ gives an approximation of the integral.

\subsubsection{Field distributions}

The SIE method also offers the possibility to calculate the electromagnetic field in the near-field of the studied object. Figure 5 (b) shows the electric field intensity around a rectangular gold dipole antenna. The antenna's arms are $40 \times 40 \times 100 \mathrm{~nm}^{3}$ large, rounded to a radius of $5 \mathrm{~nm}$ and have a gap of $20 \mathrm{~nm}$. The dielectric function for gold was taken from Ref. 45 and cross section calculation revealed a scattering resonance at $660 \mathrm{~nm}$, at which Figs. $5(\mathrm{~b}, \mathrm{c})$ are shown. Figure $5(\mathrm{c})$ is a magnified image of the gap between the two antenna arms, clearly showing the smooth field distribution. The DDA, for example, can only accurately compute the field at least one grid spacing from the surface ${ }^{46}$ before the singular behavior of the discretization elements begins to distort the field. In the FDTD method, the fields are only stored at certain points in the grid cells, again introducing errors, predominately at rounded edges, where staircasing occurs. In the SIE method, however, one can see that the field shows no trace of discretization artifacts, even much closer to the surface than the triangles' sizes (Fig. 5 (a). With an intensity enhancement factor of approximately 1000 in the gap between the metallic arms and even 

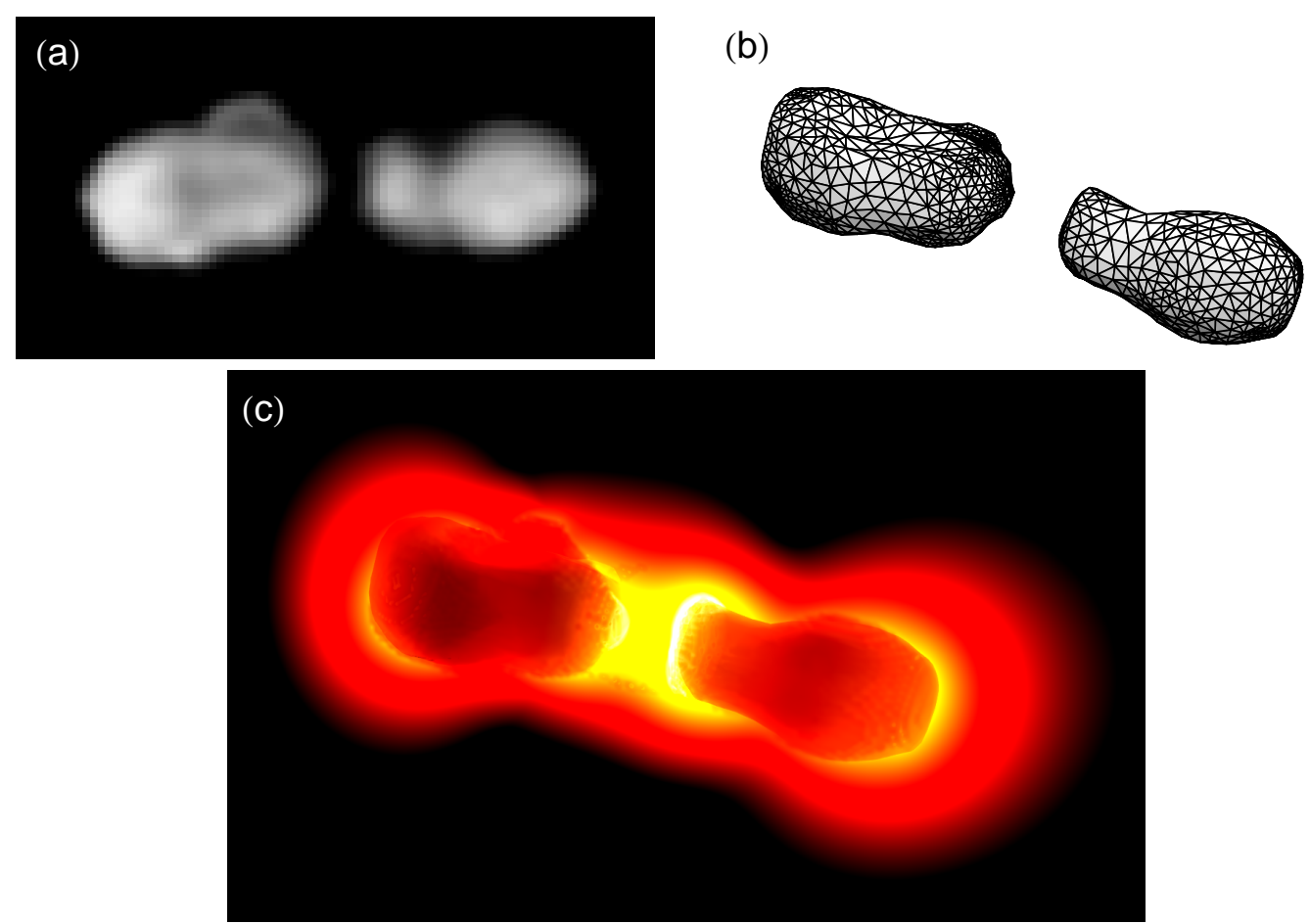

Figure 6. Simulation of a realistic dipole antenna with approx. $100 \mathrm{~nm}$ arm length. Subfigure (a) shows the micrograph which was used to recreate a $3 \mathrm{D}$ object (b). The near-field intensity distribution is shown in (c). The color scale in (c) is similar to that in Fig. 5, but the transparent representation inhibits quantitative analysis.

more at their inner corners, it is clear that the given geometry represents a resonant condition, displaying the SIE method's suitability for simulating plasmonic structures in resonance conditions.

\subsubsection{Asymmetric and nonregular scatterers}

A great strength of the SIE formulation is the surface meshing approach which proves to be much more flexible than the cubic volume discretization used, for example, in many VIE methods. As shown in Fig. 1, an unstructured mesh such as the triangular surface discretization used in the presented approach can much more accurately approximate rounded and nonregular contours. In addition, as one expects the electromagnetic field distribution to vary more rapidly near sharp edges than around flat surfaces, the unstructured grid used can easily be refined here, keeping computational costs to a minimum while retaining accuracy. Also, it has been shown that sharp-edged objects exhibit different spectral properties than similar objects with rounded edges, ${ }^{47}$ the former of which one does not expect to encounter on the nanoscale. Modeling rounded edges with cubic volume approximation, however, is extremely cumbersome while it can be easily and efficiently done with surface meshing.

Using these flexible meshing possibilities, one can greatly widen the range of simulated systems. One is no longer restricted to idealized shapes like cubes, spheres and ellipsoids, but one can study the optical properties of realistic nanostructures like one might find in a laboratory. Figure 6 (a) shows a micrograph of a dipole antenna produced by electron-beam lithography. ${ }^{48}$ Using this image as a height map, one is able to create a 3D approximation of the original structure. Meshing this 3D object (Fig. 6 (b), and assuming a gold dielectric function from Ref. 45, one is then able to compute its near-field response. Figure 6 (c) shows the intensity distribution upon lateral illumination polarized along the antenna's axis at the wavelength of scattering resonance. The color scale of this image is similar to that in Fig. 5, but the transparent representation inhibits quantitative analysis. Nonetheless, one can clearly see the localized field enhancement, which looks quite different from that which one would expect from a more regular structure such as in Fig. 5. Returning to the far-field, the scattering spectra of the nonregular and the ideal dipole are nearly identical (not shown). This shows that the field in the 
close proximity of the antennae contains components that are not dipolar in nature and thus can vary without having an influence on the antennae's far-field properties.

\section{CONCLUSION}

In this paper, we have presented a method for numerical simulation of Maxwell's equations based on a surface integral equation approach. A brief introduction to other popular simulation routines shows that the SIE formulation possesses a range of capabilities not inherent to other approaches, notably flexible surface meshing as well as excellent near- and far-field accuracy. Useful post-processing routines exploiting these capabilities were presented, demonstrating the versatility of the SIE approach. Simulations of two plasmonic systems show that the chosen SIE/PMCHW approach does not suffer from resonance instabilities and thus presents an apt technique for simulations in the domain of plasmonics.

A particular talent of the presented SIE method is the calculation of extreme near-field properties of irregular objects. We have shown simulations of a realistic gold nanoantenna and the resulting near-field distribution. While the far-field properties of such structures might not be directly influenced by their microscopic geometry, the near-field intensity distribution depends sensitively on a particle's symmetry and surface composition. The potential to calculate this influence makes the SIE method a valuable tool for studying the effects of local field enhancement on Raman scattering and fluorescence of single molecules.

\section{REFERENCES}

[1] Purcell, E. M. and Pennypacker, C. R., "Scattering and absorption of light by nonspherical dielectric grains," Astrophys. J. 186, 705-714 (1973).

[2] Draine, B. T., "The discrete-dipole approximation and its application to interstellar graphite grains," Astrophys. J. 333, 848-872 (1988).

[3] Draine, B. T. and Flatau, P. J., "Discrete-dipole approximation for scattering calculations," J. Opt. Soc. Am. A 11(4), 1491-1499 (1994).

[4] Nieto-Vesperinas, M., [Scattering and Diffraction in Physical Optics], World Scientific, 2 ed. (2006).

[5] Yang, W.-H. Y., Schatz, G. C., and Van Duyne, R. P., "Discrete dipole approximation for calculating extinction and raman intensities for small particles with arbitrary shapes," J. Comput. Phys. 103(3), 869875 (1995).

[6] Averitt, R. D., Westcott, S. L., and Halas, N. J., "Linear optical properties of gold nanoshells," J. Opt. Soc. Am. B 16(10), 1824-1832 (1999).

[7] Jensen, T. R., Schatz, G. C., and Van Duyne, R. P., "Nanosphere lithography: Surface plasmon resonance spectrum of a periodic array of silver nanoparticles by ultraviolet-visible extinction spectroscopy and electrodynamic modeling," J. Phys. Chem. B 103(13), 2394-2401 (1999).

[8] Hao, E., Li, S., Bailey, R. C., Zou, S., Schatz, G. C., and Hupp, J. T., "Optical properties of metal nanoshells," J. Phys. Chem. B 108(4), 1224-1229 (2004).

[9] Saiz, J. M., Valle, P. J., González, F., Ortiz, E. M., and Moreno, F., "Scattering by a metallic cylinder on a substrate: burying effects," Opt. Lett. 21(17), 1330 (1996).

[10] Valle, P. J., Moreno, F., and Saiz, J. M., "Comparison of real- and perfect-conductor approaches for scattering by a cylinder on a flat substrate," J. Opt. Soc. Am. A 15(1), 158 (1998).

[11] Martin, O. J. F. and Piller, N. B., "Electromagnetic scattering in polarizable backgrounds," Phys. Rev. E 58(3), 3909-3915 (1998).

[12] Kern, A. M. and Martin, O. J. F., "Surface integral formulation for 3D simulations of plasmonic and high permittivity nanostructures," J. Opt. Soc. Am. A 26(4), 732-740 (2009).

[13] Song, J., Lu, C., Chew, W., and Lee, S., "Fast illinois solver code (fisc)," IEEE Trans. Antennas Propag. 40(3), 27-34 (1998).

[14] Neilson, J. M. and Bunger, R., "Surface integral equation analysis of quasi-optical launchers," IEEE Trans. Plasma Sci. 30(3), 794-799 (2002).

[15] Chu, Y.-H. and Chew, W. C., "Large-scale computation for electrically small structures using surfaceintegral equation method," Microwave Opt. Tech. Lett. 47(6), 525-530 (2005). 
[16] Jung, J. and Sondergaard, T., "Green's function surface integral equation method for theoretical analysis of scatterers close to a metal interface," Phys. Rev. B 77(24), 245310 (2008).

[17] García de Abajo, F. J. and Howie, A., "Retarded field calculation of electron energy loss in inhomogeneous dielectrics," Phys. Rev. B 65(11), 115418 (2002).

[18] Romero, I., Aizpurua, J., Bryant, G. W., and García de Abajo, F. J., "Plasmons in nearly touching metallic nanoparticles: singular response in the limit of touching dimers," Opt. Express 14(21), 9988-9999 (2006).

[19] Yee, K., "Numerical solution of inital boundary value problems involving maxwell's equations in isotropic media," IEEE Trans. Antennas Propag. 14(3), 302-307 (1966).

[20] Taflove, A. and Brodwin, M. E., "Numerical solution of steady-state electromagnetic scattering problems using the time-dependent maxwell's equations," IEEE Trans. Microwave Theory Tech. 23(8), 623-630 (1975).

[21] Kunz, K. S. and Simpson, L., "A technique for increasing the resolution of finite-difference solutions of the Maxwell equation," IEEE Trans. Electromagn. Compat. EMC-23(4), 419-422 (1981).

[22] Kern, A. and Walther, M., "Subgridding in the finite-difference time-domain method for simulating the interaction of terahertz radiation with metal," J. Opt. Soc. Am. B 25(3), 279-285 (2008).

[23] Monk, P., [Finite element methods for Maxwell's equations], Oxford University Press (2003).

[24] Mur, G., "Absorbing boundary conditions for the finite-difference approximation of the time-domain electromagnetic-field equations," IEEE Trans. Electromagn. Compat. EMC-23(4), 377-382 (1981).

[25] Berenger, J.-P., "A perfectly matched layer for the absorption of electromagnetic waves," J. Comput. Phys. 114, 185-200 (1994).

[26] Paulus, M. and Martin, O. J. F., "Light propagation and scattering in stratified media: a green's tensor approach," J. Opt. Soc. Am. A 18(4), 854-861 (2001).

[27] Chew, W. C., [Waves and Fields in Inhomogeneous Media], Van Nostrand Reinhold (1990).

[28] Harrington, R. F., [Field Computation by Moment Methods], Macmillan (1968).

[29] Rao, S., Wilton, D., and Glisson, A., "Electromagnetic scattering by surfaces of arbitrary shape," IEEE Trans. Antennas Propag. 30(3), 409-418 (1982).

[30] Sheng, X. Q., Jin, J. M., Song, J., Chew, W. C., and Lu, C. C., "Solution of combined-field integral equation using multilevel fast multipole algorithm for scattering by homogeneous bodies," IEEE Trans. Antennas Propag. 46(11), 1718-1726 (1998).

[31] Ylä-Oijala, P., "Application of a novel CFIE for electromagnetic scattering by dielectric objects," Microwave Opt. Tech. Lett. 35(1), 3-5 (2002).

[32] Ylä-Oijala, P. and Taskinen, M., "Application of combined field integral equation for electromagnetic scattering by dielectric and composite objects," IEEE Trans. Antennas Propag. 53(3), 1168-1173 (2005).

[33] Poggio, A. J. and Miller, E. K., "Integral equation solutions of three dimensional scattering problems," in [Computer Techniques for Electromagnetics], Permagon, Oxford, U.K. (1973).

[34] Chang, Y. and Harrington, R., "A surface formulation for characteristic modes of material bodies," IEEE Trans. Antennas Propag. 25(6), 789-795 (1977).

[35] Wu, T.-K. and Tsai, L. L., "Scattering from arbitrarily-shaped lossy dielectric bodies of revolution," Radio Science 12, 709-718 (1977).

[36] Medgyesi-Mitschang, L. N., Putnam, J. M., and Gedera, M. B., "Generalized method of moments for three-dimensional penetrable scatterers," J. Opt. Soc. Am. A 11(4), 1383-1398 (1994).

[37] Graglia, R. D., "On the numerical integration of the linear shape functions times the 3-d green's function or its gradient on a plane triangle," IEEE Trans. Antennas Propag. 41(10), 1448-1455 (1993).

[38] Ylä-Oijala, P. and Taskinen, M., "Calculation of CFIE impedance matrix elements with RWG and $\mathrm{n} \times$ RWG functions," IEEE Trans. Antennas Propag. 51(8), 1837-1846 (2003).

[39] Hänninen, I., Taskinen, M., and Sarvas, J., "Singularity subtraction integral formulae for surface integral equations with RWG, rooftop and hybrid basis functions," Progress In Electromagnetics Research PIER 63, 243-278 (2006).

[40] Kottmann., J. P. and Martin, O. J. F., "Accurate solution of the volume integral equation for highpermittivity scatterers," IEEE Trans. Antennas Propag. 48(11), 1719-1726 (2000).

[41] Cowper, G. R., "Gaussian quadrature formulas for triangles," Int. J. Numer. Methods Eng. 7(3), 405-408 (1973). 
[42] Bohren, C. and Huffmann, D., [Absorption and scattering of light by small particles], Wiley (1983).

[43] Mie, G., "Beiträge zur optik trüber medien, speziell kolloidaler metallösungen," Ann. Phys. 25(3), 377-445 (1908).

[44] Paterson, M. S. and Yao, F. F., "Efficient binary space partitions for hidden-surface removalefficient binary space partitions for hidden-surface removal and solid modeling," Discrete and Computational Geometry 5(1), 485 - 503 (1990).

[45] Johnson, P. B. and Christy, R. W., "Optical constants of the noble metals," Phys. Rev. B 6(12), 4370-4379 (1972).

[46] Draine, B. T. and Flatau, P. J., "Discrete-dipole approximation for periodic targets: theory and tests," J. Opt. Soc. Am. A 25(11), 2693 (2008).

[47] Davis, L. C., "Electostatic edge modes of a dielectric wedge," Phys. Rev. B 14(12), 5523-5525 (1976).

[48] Fischer, H. and Martin, O. J. F., "Fabrication and optical characterization of plasmonic nanoantennas," Appl. Phys. Lett., submitted (2009). 